PROCEEDINGS OF THE

AMERICAN MATHEMATICAL SOCIETY

Volume 132, Number 2, Pages 607-615

S 0002-9939(03)07062-X

Article electronically published on June 30, 2003

\title{
ON THE HARMONIC HOPF CONSTRUCTION
}

\author{
ANDREAS GASTEL \\ (Communicated by Bennett Chow)
}

\begin{abstract}
The harmonic Hopf construction is an equivariant ansatz for harmonic maps between Euclidean spheres. We prove existence of solutions in the case that has been open. Moreover, we show that the harmonic Hopf construction on every bi-eigenmap with at least one large eigenvalue has a countable family of solutions (if it has one).
\end{abstract}

\section{INTRODUCTION}

The harmonic Hopf construction is an equivariant ansatz for harmonic maps between Euclidean spheres. It was invented by Smith in his thesis [S1. The data to start with are harmonic bi-eigenmaps. Given a compact Riemannian manifold $M$, a map $f: M \rightarrow S^{k}$ is called harmonic if it is a smooth critical point of the energy $E(f):=\frac{1}{2} \int_{M}|D f|^{2} d \mathrm{vol}_{M}$ or equivalently if it solves the system of differential equations

$$
\Delta f+|D f|^{2} f=0 .
$$

A harmonic map $f: S^{m-1} \rightarrow S^{n-1}(m, n \geq 2)$ is called an eigenmap if $|D f|^{2} \equiv \lambda$ is constant. It is well-known that $f$ is a harmonic eigenmap if and only if its components are harmonic polynomials of common degree $\mu$, in which case $\lambda=$ $\mu(m+\mu-2)$. The integer $\lambda$ is called the eigenvalue of $f$.

Finally, for $m_{1}, m_{2}, n \geq 2$, a harmonic map $f: S^{m_{1}-1} \times S^{m_{2}-1} \rightarrow S^{n-1}$ is called a bi-eigenmap if each of the restrictions $f\left(\cdot, x_{2}\right)$ for every $x_{2} \in S^{m_{2}-1}$ and $f\left(x_{1}, \cdot\right)$ for every $x_{1} \in S^{m_{1}-1}$ is a harmonic eigenmap. The corresponding eigenvalues $\lambda_{1}$ and $\lambda_{2}$ are also called the eigenvalues of $f$. For example, every orthogonal multiplication $\mathbb{R}^{m_{1}} \times \mathbb{R}^{m_{2}} \rightarrow \mathbb{R}^{n}$, restricted to the unit spheres, gives a harmonic bi-eigenmap with eigenvalues $m_{1}-1$ and $m_{2}-1$. See [ER, ch. VIII] for a detailed exposition of (bi-)eigenmaps.

Topologically, the Hopf construction on a map $f: S^{m_{1}-1} \times S^{m_{2}-1} \rightarrow S^{n-1}$ is the map $h_{f}: S^{m_{1}+m_{2}-1} \rightarrow S^{n}$ given by

$$
h_{f}\left(x_{1} \sin s, x_{2} \cos s\right):=\left(f\left(x_{1}, x_{2}\right) \sin 2 s, \cos 2 s\right)
$$

where we write $x \in S^{m_{1}+m_{2}-1}$ uniquely (apart from a null set) as $\left(x_{1} \sin s, x_{2} \cos s\right.$ ) with $x_{1} \in S^{m_{1}-1}, x_{2} \in S^{m_{2}-1}, s \in[0, \pi / 2]$. This Hopf construction is a tool for constructing homotopically nontrivial maps between spheres of large dimensions. The aim now is to modify the construction in such a way that it produces a harmonic

Received by the editors July 12, 2001 and, in revised form, September 27, 2002.

2000 Mathematics Subject Classification. Primary 58E20.

(C)2003 American Mathematical Society 
representative of the homotopy class of the Hopf map. It was proved by Smith S2] that the ansatz

$$
h\left(x_{1} \sin s, x_{2} \cos s\right)=\left(f\left(x_{1}, x_{2}\right) \sin \alpha(s), \cos \alpha(s)\right)
$$

with some function $\alpha:[0, \pi / 2] \rightarrow[0, \pi]$ produces a harmonic map homotopic to $h_{f}$ if $f$ is a harmonic bi-eigenmap with eigenvalues $\lambda_{1}, \lambda_{2}$ and $\alpha$ satisfies the ordinary differential equation

$$
\alpha^{\prime \prime}+\left(\left(m_{1}-1\right) \cot s-\left(m_{2}-1\right) \tan s\right) \alpha^{\prime}-\left(\frac{\lambda_{1}}{\sin ^{2} s}+\frac{\lambda_{2}}{\cos ^{2} s}\right) \sin \alpha \cos \alpha=0
$$

with the boundary values $\alpha(0)=0, \alpha(\pi / 2)=\pi$. Therefore, the natural question arises, for which harmonic bi-eigenmap $f$ this degenerate boundary value problem has a solution. Here we collect the results that have been achieved so far:

- Smith [S2] proved that in the case $m_{1}=m_{2}=2$ a harmonic Hopf construction exists if and only if $\lambda_{1}=\lambda_{2}$.

- Ratto R2] proved that in the case $m_{1} \geq 3, m_{2}=2$, no harmonic Hopf construction can exist if $\lambda_{1} \leq\left(m_{1}-1\right) \lambda_{2}$.

- For the case $m_{1}, m_{2} \geq 3$ Ratto proved [R1] that there is a harmonic Hopf construction if $4 \lambda_{1}<\left(m_{1}-2\right)^{2}$ and $4 \lambda_{2}<\left(m_{2}-2\right)^{2}$, or if $m_{1}=m_{2}$ and $\lambda_{1}=\lambda_{2}$.

- The latter result was improved by Ding $[\mathrm{D}$ who proved that there is always a harmonic Hopf construction in the case $m_{1}, m_{2} \geq 3$, without restriction on the eigenvalues.

- A different (somewhat simpler) proof of Ding's theorem was given by the author in [G].

Thus, the only case that remains open is $m_{1} \geq 3, m_{2}=2$, and $\lambda_{1}>\left(m_{1}-1\right) \lambda_{2}$. One objective of this note is to fill this gap by proving that for a harmonic bi-eigenmap with dimensions and eigenvalues in this range a harmonic Hopf construction always exists. This is the statement of Theorem 3.1.

By a similar proof, we can also show that, in the case $m_{1}, m_{2} \geq 3$, there are actually countably many solutions to the harmonic Hopf construction on any eigenmap for which $4 \lambda_{1}>\left(m_{1}-2\right)^{2}$ or $4 \lambda_{2}>\left(m_{2}-2\right)^{2}$ holds. This is stated in Theorem 4.1 The phenomenon of having many solutions resembles very much the the situation for rotationally symmetric harmonic maps $B^{m} \rightarrow S^{m}$ [JK], respectively $S^{m} \rightarrow S^{m}$ $\mathrm{BC}$, where in dimensions $3 \leq m \leq 6$ one has infinitely many solutions. Our proof uses some ideas from $[\mathrm{BC}$, which have to be modified, however, because here the situation is less symmetric.

In using a shooting method, our proof here is closer to Ratto's existence proof than to Ding's, who argued by minimizing certain energies. However, while Ratto (and also the author in [G]) uses some kind of "two-sided shooting", in the current paper we shoot from one of the degenerate ends of the interval. This results in a less technical proof and could also be used to again re-prove Ding's existence theorem in the cases not covered here.

After submitting this paper, I received the preprint [DFL] where Theorem 3.1 is proved independently by different methods.

\section{Preliminaries}

We will use the notation $\varphi(\infty)$ for $\lim _{t \rightarrow \infty} \varphi(t)$ and $\varphi(-\infty)$ for $\lim _{t \rightarrow-\infty} \varphi(t)$. 
It is convenient to make the transformation $\varphi(t)=\alpha\left(\arctan e^{t}\right)-\pi / 2$, which transforms (1.1) into the equation

$$
\varphi^{\prime \prime}(t)+\frac{\left(m_{1}-2\right) e^{-t}-\left(m_{2}-2\right) e^{t}}{e^{-t}+e^{t}} \varphi^{\prime}(t)+\frac{\lambda_{1} e^{-t}+\lambda_{2} e^{t}}{e^{-t}+e^{t}} \sin \varphi(t) \cos \varphi(t)=0,
$$

while the above-mentioned boundary value problem becomes

$$
\text { (2.1) with } \varphi(-\infty)=-\frac{\pi}{2} \text { and } \varphi(\infty)=\frac{\pi}{2} \text {. }
$$

Writing

$$
I(t):=\int_{0}^{t} \frac{\left(m_{1}-2\right) e^{-\tau}-\left(m_{2}-2\right) e^{\tau}}{e^{-\tau}+e^{\tau}} d \tau-\frac{m_{1}-2}{2} \log 2,
$$

we have the divergence form of (2.1):

$$
\frac{d}{d t}\left[e^{I(t)} \varphi^{\prime}(t)\right]+e^{I(t)} \frac{\lambda_{1} e^{-t}+\lambda_{2} e^{t}}{e^{-t}+e^{t}} \sin \varphi(t) \cos \varphi(t)=0 .
$$

Multiplying by $e^{I(t)} \varphi^{\prime}(t)$ and abbreviating

$$
\begin{aligned}
a(t) & :=e^{2 I(t)} \frac{\lambda_{1} e^{-t}+\lambda_{2} e^{t}}{e^{-t}+e^{t}}, \\
W(\varphi, t) & :=e^{2 I(t)} \varphi^{\prime}(t)^{2}+a(t) \sin ^{2} \varphi(t),
\end{aligned}
$$

we get a reformulation of (2.1) which will turn out to be the key for its understanding:

$$
W^{\prime}(\varphi, t)=a^{\prime}(t) \sin ^{2} \varphi(t) .
$$

If $m_{1} \geq 3$, every solution $\varphi$ of the boundary value problem (2.2) satisfies

$$
W(\varphi,-\infty)=a(-\infty)=0, \quad W(\varphi, \infty)=a(\infty),
$$

where the latter equals 0 if $m_{2} \geq 3$, and $\lambda_{2}$ if $m_{2}=2$.

The following lemma defines "well-posed" initial value problems. We let

$$
\mu:=\frac{1}{2}\left[-\left(m_{1}-2\right)+\sqrt{\left(m_{1}-2\right)^{2}+4 \lambda_{1}}\right],
$$

which is in $\mathbb{N}$ because $\lambda_{1}$ is of the form $\lambda_{1}=\mu\left(m_{1}+\mu-2\right)$.

Lemma 2.1. Assume $m_{1} \geq 3$. For every $b \geq 0$ there is a unique solution $\varphi_{b}: \mathbb{R} \rightarrow$ $\mathbb{R}$ of (2.1) that satisfies $\varphi_{b}(t) \simeq-\pi / 2+b e^{\mu t}$ for $t \rightarrow-\infty$. The functions $\varphi_{b}$ and $\varphi_{b}^{\prime}$ (and therefore all derivatives of $\varphi_{b}$ ) depend continuously on $b$.

Proof. We consider $b>0$ fixed and rewrite (2.1) as $L \varphi=0$. For $t \ll-1$ we compute

$$
L\left(-\frac{\pi}{2}+b e^{\mu t} \pm e^{(\mu+1) t}\right)= \pm\left(2 \mu+m_{1}-1\right) e^{(\mu+1) t}+O\left(e^{(\mu+2) t}\right),
$$

which implies existence of $q<0$ (depending continuously on $b$ ) such that

$$
\begin{array}{ll}
L \varphi_{+}>0 \text { in }(-\infty, q) & \text { for } \varphi_{+}:=-\frac{\pi}{2}+b e^{\mu t}+e^{(\mu+1) t}, \\
L \varphi_{-}<0 \text { in }(-\infty, q) & \text { for } \varphi_{-}:=-\frac{\pi}{2}+b e^{\mu t}-e^{(\mu+1) t} .
\end{array}
$$

For $k \in \mathbb{N}$ with $k>|q|$ we define $p_{k}:(-\infty, q) \rightarrow \mathbb{R}$ by

$$
\begin{aligned}
& p_{k}(t):=-\frac{\pi}{2}+b e^{\mu t} \text { for } t \leq-k, \\
& L p_{k}=0 \text { in }(-k, q) \text { with } p_{k}(-k)=-\frac{\pi}{2}+b e^{-\mu k} \text { and } p_{k}^{\prime}(-k)=\mu b e^{-\mu k} .
\end{aligned}
$$


For $h_{+}:=\varphi_{+}-p_{k}$ we have $h_{+}(-k)>0$ and $h_{+}^{\prime}(-k)>0$, and as long as $h_{+} \geq 0$, (2.6) implies that $h_{+}^{\prime \prime}(t)+D(t) h_{+}^{\prime}(t)>0$, hence $h_{+}$cannot have a local maximum there. The same applies to $h_{-}:=p_{k}-\varphi_{-}$. Therefore $h_{+}$and $h_{-}$are monotonically increasing, and we have shown

$$
\begin{aligned}
& \varphi_{-}(t) \leq p_{k}(t) \leq \varphi_{+}(t), \\
& \varphi_{-}^{\prime}(t) \leq p_{k}^{\prime}(t) \leq \varphi_{+}^{\prime}(t)
\end{aligned}
$$

for all $t<q$ and all $k>|q|$. This means that the $p_{k}$ are uniformly bounded in $C^{1}((-\infty, q))$, hence in $C^{\infty}((-\infty, q))$, and converge to a solution $\varphi_{b}$ of (2.1) on $(-\infty, q)$ as $k \rightarrow \infty$. This solution can be continued to all of $\mathbb{R}$, which shows the existence part of the lemma. by

To prove uniqueness, we consider $\varphi_{b}$ as constructed above and define $\eta_{s}$ for $s \in \mathbb{R}$

For $t \ll-1$ we have

$$
\eta_{s}(t):=\varphi_{b}(t)+s\left(e^{\mu t}+e^{(\mu+1) t}\right) .
$$

$$
L \eta_{s}(t)=s\left(2 \mu+m_{1}-1\right) e^{(\mu+1) t}+s O\left(e^{(\mu+2) t}\right) .
$$

Therefore there exists $r<0$ such that for $t \leq r$

$$
L \eta_{s}(t)<0 \text { for all } s \in(-1,0), \quad L \eta_{s}(t)>0 \text { for all } s \in(0,1) .
$$

The argument from the proof of (2.8) and (2.9) is now valid with $\varphi_{-}$and $\varphi_{+}$ replaced by $\eta_{-s}$ and $\eta_{s}$ for arbitrarily small $s>0$. This implies that every solution $\varphi$ of (2.1) with the same asymptotics at $-\infty$ as $\varphi_{b}$ lies between $\eta_{-s}$ and $\eta_{s}$ for arbitrarily small $s>0$, which means $\varphi=\varphi_{b}$ on $(-\infty, r)$ and therefore on all of $\mathbb{R}$.

Continuous dependence on $b$ is shown the same way: on $(-\infty, r)$ we have for $\varepsilon>0$ small that $\eta_{-\varepsilon}<\varphi_{b+\beta}<\eta_{\varepsilon}$ and $\eta_{-\varepsilon}^{\prime}<\varphi_{b+\beta}^{\prime}<\eta_{\varepsilon}^{\prime}$ for all $|\beta|<\varepsilon$. This completes the proof of Lemma 2.1, up to the case $b=0$ which is even simpler.

\section{THE CASE $m_{2}=2$}

We consider the harmonic Hopf construction with $m_{2}=2, m_{1}>2$. Ratto [R2] proved that there is no solution if $\lambda_{1} \leq\left(m_{1}-1\right) \lambda_{2}$. We will show that otherwise a solution exists. Doing so, at the same time we get a simple proof of Ratto's nonexistence result.

Theorem 3.1. Assume $m_{1} \geq 3$ and $m_{2}=2$. The boundary value problem (2.2) (and hence the harmonic Hopf construction) has a solution if and only if $\lambda_{1}>$ $\left(m_{1}-1\right) \lambda_{2}$.

Proof. If $m_{2}=2, a$ is easily integrated:

$$
a(t)=\frac{\lambda_{2}+\lambda_{1} e^{-2 t}}{\left(1+e^{-2 t}\right)^{m_{1}-1}} .
$$

We compute

$$
a^{\prime}(t)=\frac{2 e^{-2 t}}{\left(1+e^{-2 t}\right)^{m_{1}}}\left[\left(m_{1}-1\right) \lambda_{2}-\lambda_{1}+\left(m_{1}-2\right) \lambda_{1} e^{-2 t}\right] .
$$

If $\lambda_{1} \leq\left(m_{1}-1\right) \lambda_{2}$, we have $a^{\prime}>0$ on $\mathbb{R}$. Integrating (2.4), we see that (2.5) can only be valid if $\sin ^{2} \varphi \equiv 1$; hence there is no solution of (2.2).

In the case $\lambda_{1}>\left(m_{1}-1\right) \lambda_{2}$ we need the following two lemmas. In Lemma 3.2 we also include the case $m_{2} \geq 3$ for later use. 
Lemma 3.2. Assume $m_{2}=2$ and $\lambda_{1}>\left(m_{1}-1\right) \lambda_{2}$, or $m_{2} \geq 3$. If $b \ll 1$, the solution $\varphi_{b}$ increases monotonically up to $T_{b}$ with $\varphi_{b}\left(T_{b}\right)=\pi / 2$.

Proof. In the $m_{2}=2$ case, we see from (3.1) that $a^{\prime}\left(t_{0}\right)=0$ for exactly one $t_{0} \in \mathbb{R}$, and $a^{\prime}<0$ on $\left(t_{0}, \infty\right)$. If $m_{2} \geq 3$, since $a$ is positive and not oscillating with $a(\infty)=0$, we also find $t_{0} \in \mathbb{R}$ such that $a^{\prime}<0$ on $\left(t_{0}, \infty\right)$. We will prove the following assertion: For every $b \ll 1$ there exists $t_{1}>t_{0}$ such that

$$
\varphi_{b}^{\prime}>0 \text { on }\left(-\infty, t_{1}\right) \quad \text { and } \quad \int_{-\infty}^{t_{1}} a^{\prime}(t) \cos ^{2} \varphi_{b}(t) d t<0 .
$$

Integrating (2.4) and using (2.5), we see that the latter inequality implies

$$
W\left(\varphi_{b}, t_{1}\right)=a\left(t_{1}\right)-\int_{-\infty}^{t_{1}} a^{\prime}(t) \cos ^{2} \varphi_{b}(t) d t>a\left(t_{1}\right),
$$

from which we infer by (2.4) that $W\left(\varphi_{b}, t\right) \geq a(t)+\varepsilon$ for some $\varepsilon>0$ and all $t>t_{1}$. From this we have a positive lower bound for $\varphi_{b}^{\prime}$ on $\left(t_{1}, \infty\right)$, which implies the lemma. Therefore it is sufficient to prove (3.2).

For $b>0$, we consider the functions $\eta_{b}: \mathbb{R} \rightarrow \mathbb{R}$ given by

$$
\eta_{b}(t):=\frac{1}{b}\left(\varphi_{b}(t)+\frac{\pi}{2}\right) .
$$

Note that $\eta_{b} \simeq e^{\mu t}$ as $t \rightarrow-\infty$, and $\eta_{b}$ solves the differential equation

$$
\eta^{\prime \prime}(t)+\frac{\left(m_{1}-2\right) e^{-t}-\left(m_{2}-2\right) e^{t}}{e^{-t}+e^{t}} \eta^{\prime}(t)-\frac{\lambda_{1} e^{-t}+\lambda_{2} e^{t}}{e^{-t}+e^{t}} \frac{1}{b} \sin (b \eta(t)) \cos (b \eta(t))=0 .
$$

The functions $\eta_{b}$ for $b \in(0,1]$ are uniformly bounded in $C^{1}$ (hence also in $C^{\infty}$ by the differential equation) on every interval $(-\infty, T)$, therefore (using also uniqueness similar to Lemma 2.1 we find that $\eta(t):=\lim _{b \backslash 0} \eta_{b}(t)$ is well-defined for all $t \in \mathbb{R}$, it satisfies

$$
\eta(t) \simeq e^{\mu t} \text { as } t \rightarrow-\infty
$$

and

$$
\eta^{\prime \prime}(t)+\frac{\left(m_{1}-2\right) e^{-t}-\left(m_{2}-2\right) e^{t}}{e^{-t}+e^{t}} \eta^{\prime}(t)-\frac{\lambda_{1} e^{-t}+\lambda_{2} e^{t}}{e^{-t}+e^{t}} \eta(t)=0,
$$

which is the limit of the differential equation (3.4) for $b \searrow 0$. Furthermore, since the $\varphi_{b}$ are convex on increasing intervals as $b \searrow 0$, we have

$$
\eta^{\prime}(t)>0 \text { for all } t \in \mathbb{R} \text {. }
$$

The differential equation (3.6) is linear and has a fundamental system consisting of two solutions with the asymptotics $\simeq e^{\nu_{-} t}$ and $\simeq e^{\nu_{+} t}$ for $t \rightarrow \infty$ where

$$
\nu_{ \pm}:=\frac{1}{2}\left[m_{2}-2 \pm \sqrt{\left(m_{2}-2\right)^{2}+4 \lambda_{2}}\right] \in \mathbb{Z} .
$$

Because of (3.7), it follows that

$$
\eta(t) \simeq c e^{\nu+t} \text { as } t \rightarrow \infty
$$

for some constant $c>0$. By (3.5), (3.8), $a^{\prime}(t) \simeq c e^{2\left(m_{1}-2\right) t}$ for $t \rightarrow-\infty$, and $a^{\prime}(t) \simeq c e^{-2\left(m_{2}-2\right) t}$ for $t \rightarrow \infty\left(\right.$ and $a^{\prime}(t) \simeq c e^{-2 t}$ if $\left.m_{2}=2\right)$, we have

$$
\lim _{t_{1} \rightarrow \infty} \int_{-\infty}^{t_{1}} a^{\prime}(t) \eta(t)^{2} d t=-\infty
$$


Using this and (3.7), we find $t_{1}>t_{0}$ for which (3.2) holds, and hence the assertion of the lemma.

Lemma 3.3. Assume $m_{2}=2$ and $\lambda_{1}>\left(m_{1}-1\right) \lambda_{2}$. If $b \gg 1$, we have $\varphi_{b}(\mathbb{R}) \subseteq$ $(-\pi / 2, \pi / 2)$.

Proof. Integrating (2.4) from $-\infty$ to $s \leq t_{0}$, using $a^{\prime}>0$ there, we observe that $W\left(\varphi_{b}, s\right)<a(s)$ and therefore

$$
\varphi_{b}\left(\left(-\infty, t_{0}\right]\right) \subseteq(-\pi / 2, \pi / 2) .
$$

Let us denote by $\psi: \mathbb{R} \rightarrow \mathbb{R}$ the solution of

$$
\psi^{\prime \prime}(t)+\left(m_{1}-2\right) \psi^{\prime}(t)+\lambda_{1} \sin \psi(t) \cos \psi(t)=0
$$

satisfying $\psi(t) \simeq-\pi / 2+e^{\mu t}$ as $t \rightarrow-\infty$. It exists uniquely, which is proved as in Lemma 2.1] Defining

$$
U(\psi, t):=\psi^{\prime}(t)^{2}+\lambda_{1} \sin ^{2} \psi(t),
$$

we infer from (3.11) that

$$
U^{\prime}(\psi, t)=-2\left(m_{1}-2\right) \psi^{\prime}(t)^{2} .
$$

Hence $U(\psi, \cdot)$ is monotonically decreasing. Since it is also bounded from below by 0 , its limit for $t \rightarrow \infty$ exists, which can be only 0 by (3.11) and (3.12):

$$
U(\psi, \infty)=0 .
$$

The functions $\psi_{b}(t):=\varphi_{b}\left(t-\mu^{-1} \log b\right)$ satisfy $\psi_{b}(t) \simeq-\pi / 2+e^{\mu t}$ as $t \rightarrow-\infty$ for all $b>0$. All $\psi_{b}$ with $b>1$ are uniformly bounded with all their derivatives on compact intervals, hence sequences $\psi_{b_{n}}$ converge on compact intervals. Uniqueness of $\psi$ with the given behavior at $-\infty$ then implies that actually $\lim _{b \rightarrow \infty} \psi_{b}(t)=\psi(t)$ for all $t \in \mathbb{R}$. Now we consider

$$
V(\varphi, t):=\varphi^{\prime}(t)^{2}+\frac{\lambda_{1} e^{-t}+\lambda_{2} e^{t}}{e^{-t}+e^{t}} \sin ^{2} \varphi(t)
$$

and calculate, using the differential equation,

$$
V^{\prime}\left(\varphi_{b}, t\right)=-\frac{2\left(m_{1}-2\right)}{1+e^{2 t}} \varphi_{b}^{\prime}(t)^{2}+\left(\frac{\lambda_{1} e^{-t}+\lambda_{2} e^{t}}{e^{-t}+e^{t}}\right)^{\prime} \sin ^{2} \varphi_{b}(t),
$$

which means $V^{\prime}\left(\varphi_{b}, t\right)<0$ on $\mathbb{R}$ because $\lambda_{1}>\lambda_{2}$. From (3.13) and $\psi_{b} \rightarrow \psi$, we find $b_{0}$ such that for every $b \geq b_{0}$ we have some $\tau_{b} \leq t_{0}$ for which $V\left(\varphi_{b}, \tau_{b}\right)<\lambda_{2}$, hence $W\left(\varphi_{b}, t_{0}\right)<\lambda_{2}$. Therefore, for $b \geq b_{0}$ we have $W\left(\varphi_{b}, t\right)<a(t)$ for all $t>t_{0}$, and the lemma follows.

Now the proof of Theorem 3.1 is quite straightforward: Let $b_{0}$ be the supremum of all $b>0$ for which $\varphi_{b}$ is monotonically increasing up to some $T_{b}$ with $\varphi_{b}\left(T_{b}\right)=\pi / 2$. This supremum is in $(0, \infty)$ because of Lemma 3.2 and Lemma 3.3. From Lemma 2.1 we know that $\varphi_{b}$ and $\varphi_{b}^{\prime}$ depend continuously on $b$, which implies that $\varphi_{b_{0}}$ is nondecreasing and bounded from above by $\pi / 2$ (which is easily seen by the supremum property of $\left.b_{0}\right)$. Hence $\varphi_{b_{0}}(\infty)$ exists. But $\pi / 2$ is the only value that $\varphi_{b_{0}}(\infty)$ can achieve, which is seen by simple inspection of (2.1). Therefore $\varphi_{b_{0}}$ is the solution we had to find to prove Theorem 3.1 


\section{Multiple solutions}

Now we consider $m_{1}, m_{2} \geq 3$, the case for which existence of harmonic Hopf constructions has already been shown by Ratto [R1] and Ding [D. We will show that for each bi-eigenmap with one sufficiently large eigenvalue, there are actually countably many solutions of the harmonic Hopf construction.

Theorem 4.1. Assume $m_{1}, m_{2} \geq 3$ and $4 \lambda_{1}>\left(m_{1}-2\right)^{2}$ or $4 \lambda_{2}>\left(m_{2}-2\right)^{2}$. Then for each odd number $k \in \mathbb{N}$ there is a solution $\phi_{k}$ of (2.2) which has exactly $k$ zeroes.

W.l.o.g. we can assume $4 \lambda_{1}>\left(m_{1}-2\right)^{2}$. Besides Lemma 3.2 from the previous section, we need the following lemma.

Lemma 4.2. Assume $m_{1}, m_{2} \geq 3$ and $4 \lambda_{1}>\left(m_{1}-2\right)^{2}$. For each $k \in \mathbb{N}$ there is $c(k)>0$ such that $\varphi_{b}$ has at least $k$ zeroes whenever $b>c(k)$. Moreover, $\varphi_{b}$ can reach $\pm \frac{\pi}{2}$ only after those zeroes.

Proof. The solution $\psi$ of (3.11) which was constructed in Lemma 3.3 converges to 0 as $t \rightarrow \infty$, and so does $\psi^{\prime}$, because of (3.13). It is standard to infer

$$
\psi(t) \simeq C_{1} e^{\left(1-m_{1} / 2\right) t} \sin \left(\omega t-C_{2}\right) \text { as } t \rightarrow \infty
$$

from this, with constants $C_{1}, C_{2} \in \mathbb{R}$ and $\omega:=\frac{1}{2} \sqrt{4 \lambda_{1}-\left(m_{1}-2\right)^{2}}$, because asymptotically $\psi$ solves $\psi^{\prime \prime}+\left(m_{1}-2\right) \psi^{\prime}+\lambda_{1} \psi=0$. As $b \rightarrow \infty$, the functions $\varphi_{b}\left(t-\mu^{-1} \log b\right)$ converge to $\psi$ in $C^{1}(\mathbb{R})$, which is proved as in Lemma 3.3. The claim now follows from the asymptotics stated in (4.1), and from the fact that $\psi$ never reaches $\pm \frac{\pi}{2}$ because of (3.12) and $U(\psi,-\infty)=\lambda_{1}$.

Proof of Theorem 4.1. We define

$$
\begin{aligned}
t_{b} & :=\inf \left\{t \in \mathbb{R}: \varphi_{b}(t)=-\pi / 2\right\}, \\
T_{b} & :=\inf \left\{t \in \mathbb{R}: \varphi_{b}(t)=\pi / 2\right\}, \\
n_{b} & :=\operatorname{card}\left\{t<\inf \left\{t_{b}, T_{b}\right\}: \varphi_{b}(t)=0\right\} .
\end{aligned}
$$

First we construct $\phi_{1}$. Let

$$
S_{1}:=\left\{b>0: n_{b}=1, T_{b}<\infty\right\} .
$$

$S_{1}$ is not empty because of Lemma 3.2 and bounded from above because of Lemma 4.2. Therefore $a_{1}:=\sup S_{1} \in(0, \infty)$ exists. Since all of the $\varphi_{b}$ for $b \in S_{1}$ are increasing, $\varphi_{a_{1}}$ is nondecreasing, but $T_{a_{1}}<\infty$ cannot hold because the same would be true for $b>a_{1}$ close to $a_{1}$. Thus $\varphi_{a_{1}}$ is bounded from above by $\pi / 2$, and $\varphi_{a_{1}}(\infty)$ exists. Again, $\pi / 2$ is the only value that (2.1) allows for $\varphi_{a_{1}}$, so $\varphi_{a_{1}}$ is a nondecreasing solution of (2.2) with one zero. The solution $\varphi_{a_{1}}$ might not be uniquely determined by these properties, so we define $b_{1}$ to be the supremum of all parameters for which the same properties hold. Then $\phi_{1}:=\varphi_{b_{1}}$ fulfills the claim of the theorem.

Now let

$$
S_{2}:=\left\{b>0: n_{b}=2, t_{b}<\infty\right\} .
$$

We want to show that $S_{2}$ is not empty. To this end we assume that $b>b_{1}$ is sufficiently close to $b_{1}$, and we will show $b \in S_{2}$. The choice of $b_{1}$ implies $n_{b} \geq 2$, because if we had $n_{b}=1$, then $\varphi_{b}$ would have to fulfill $\varphi_{b}(\infty) \in\left[0+, \frac{\pi}{2}\right)$ or have some positive local minimum, both of which are easily seen not to be possible from the differential equation (2.1). Now we can perform the argument of Lemma 3.2 
again to infer that, for $b$ close enough to $b_{1}, \varphi_{b}$ is decreasing on $\left(s_{b}, t_{b}\right]$ for some $t_{b} \in \mathbb{R}$. To do this, we do some obvious sign changes in the proof of Lemma 3.2 and replace the definition of $\eta_{b}$ by

$$
\eta_{b}(t):=\frac{\varphi_{b}(t)-\varphi_{b_{1}}(t)}{b-b_{1}}
$$

This changes the last term in (3.6) to

$$
+\frac{\lambda_{1} e^{-t}+\lambda_{2} e^{t}}{e^{-t}+e^{t}} \frac{\sin \left\{\varphi_{b_{1}}(t)+\left(b-b_{1}\right) \eta(t)\right\} \cos \left\{\varphi_{b_{1}}(t)+\left(b-b_{1}\right) \eta(t)\right\}}{b-b_{1}},
$$

which does, however, not change any of the asymptotics, because $\varphi_{b_{1}}(\infty)=\frac{\pi}{2}$. The proof goes through, therefore $n_{b}=2$, and $S_{2}$ is not empty.

Now that we know that $S_{2}$ is not empty, we can repeat what we did in the construction of $\phi_{1}$ to find a function $\phi_{2}$ with exactly two zeroes solving the boundary value problem

$$
\text { (2.1) with } \varphi(-\infty)=\varphi(\infty)=-\frac{\pi}{2} \text {. }
$$

Analogously we construct $b_{k}$ and $\phi_{k}$ for all $k \in \mathbb{N}$ by induction. For odd $k, \phi_{k}$ will solve (2.2), while for even $k$ it solves (4.2). This completes the proof of Theorem 4.1 .

Remark 4.3. In the case $m_{2}=2, m_{1} \geq 3$, there are also countably many solutions if $\lambda_{1}>\left(m_{1}-2\right) \lambda_{2}$ and $4 \lambda_{1}>\left(m_{1}-2\right)^{2}$. This can be proved by a combination of the arguments used in the proofs of the Theorems 3.1 and 4.1 .

Remark 4.4. In the case $m_{1}=m_{2}=2$, Smith [S2] proved that (2.2) is solvable iff $\lambda_{1}=\lambda_{2}$, in which case the bi-eigenmap is $f_{\mu}: S^{1} \times S^{1} \rightarrow S^{1}, f_{\mu}\left(z_{1}, z_{2}\right)=z_{1}^{\mu} z_{2}^{\mu}$, $\mu \in \mathbb{N}$. We would like to point out that in this case one can actually write down a continuous family of explicit solutions for the harmonic Hopf construction, due to the conformal invariance of energy in two dimensions: Denote by $\Sigma: S^{2} \rightarrow \mathbb{C}$ the stereographic projection and by $H: S^{3} \rightarrow S^{2}$ the Hopf fibration. Since $u \circ H$ is harmonic for every harmonic $u: S^{2} \rightarrow S^{2}$ (see [S2, Lemma 1.7]),

$$
h_{\mu, a}(x):=\Sigma^{-1}\left(a \Sigma(H(x))^{\mu}\right)
$$

is a harmonic Hopf construction on $f_{\mu}$ for every $a>0$.

\section{REFERENCES}

[BC] P. Bizoń and T. Chmaj: Harmonic maps between spheres. Proc. Royal Soc. London, Ser. A 453 (1997), 403-415. MR 98d:58039

[D] W. Y. Ding: Harmonic Hopf constructions between spheres. Internat. J. Math. 5 (1994), 849-860. MR 95k:58047

[DFL] W. Y. Ding, H. J. Fan, and J. Y. Li: Harmonic Hopf constructions between spheres II. Calc. Var. Partial Differential Equations 16 (2003), 273-282.

[ER] J. Eells and A. Ratto: Harmonic maps and minimal immersions with symmetries. Annals of Math. Studies 130, Princeton University Press, 1993. MR 94k:58033

[G] A. Gastel: Singularities of first kind in the harmonic map and Yang-Mills heat flows. Math. Z. 242 (2002), 47-62.

[JK] W. Jäger and H. Kaul: Rotationally symmetric harmonic maps from a ball into a sphere and the regularity problem for weak solutions of elliptic systems. J. Reine Angew. Math. 343 (1983), 146-161. MR 85f:58031

[R1] A. Ratto: Harmonic maps of spheres and the Hopf construction. Topology 28 (1989), 379-388. MR 90j:58035 
[R2] A. Ratto: Harmonic maps from deformed spheres to spheres. Amer. J. Math. 111 (1989), 225-238. MR 90i:58034

[S1] R. T. Smith: Harmonic mappings of spheres. Warwick thesis (1972).

[S2] R. T. Smith: Harmonic mappings of spheres. Amer. J. Math. 97 (1975), 364-385. MR 52:11949

Mathematisches Institut der Heinrich-Heine-Universität, Universitätsstr. 1, D-40225 DüsSEldorf, Germany

E-mail address: gastel@cs.uni-duesseldorf.de 